\title{
Effects of supplementation with main coffee components including caffeine and/ or chlorogenic acid on hepatic, metabolic, and inflammatory indices in patients with non-alcoholic fatty liver disease and type 2 diabetes: a randomized, double-blind, placebo-controlled, clinical trial
}

Asieh Mansour ${ }^{1}$, Mohammad Reza Mohajeri-Tehrani ${ }^{2}$, Majid Samadi ${ }^{3}$, Mostafa Qorbani ${ }^{4,5}$, Shahin Merat ${ }^{6}$, Hossein Adibi ${ }^{7}$, Hossein Poustchi ${ }^{6}$ and Azita Hekmatdoost ${ }^{{ }^{*}}$ (D)

\begin{abstract}
Background: Non-alcoholic fatty liver disease (NAFLD) is much more frequent and more severe, including cirrhosis, hepatocellular carcinoma in patients with type 2 diabetes. Coffee is a complex beverage with hundreds of compounds whereas caffeine and chlorogenic acid are the most abundant bioactive compounds. The published epidemiological data demonstrating beneficial associations between all categories of coffee exposure and ranges of liver outcomes are rapidly growing; however, the main contributors and cause-effect relationships have not yet been elucidated. To address existing knowledge gaps, we sought to determine the efficacy and safety of 6 months chlorogenic acid and/or caffeine supplementation in patients with type 2 diabetes affected by NAFLD.

Methods: This trial was carried out at two Diabetes Centers to assess the effects of supplementation with daily doses of $200 \mathrm{mg}$ chlorogenic acid, $200 \mathrm{mg}$ caffeine, $200 \mathrm{mg}$ chlorogenic acid plus $200 \mathrm{mg}$ caffeine or placebo (starch) in patients with type 2 diabetes and NAFLD. The primary endpoint was reduction of hepatic fat and stiffness measured by FibroScan, and changes in serum hepatic enzymes and cytokeratin - 18 (CK-18) levels. Secondary endpoints were improvements in metabolic (including fasting glucose, homeostasis model assessmentestimated insulin resistance (HOMA-IR), hemoglobin A1C (HBA1C), C-peptide, insulin and lipid profiles) and inflammatory (including nuclear factor k-B (NF-KB), tumor necrosis factor (TNF-a), high sensitive- $C$ reactive protein(hs-CRP)) parameters from baseline to the end of treatment.

(Continued on next page)
\end{abstract}

\footnotetext{
*Correspondence: a_hekmat2000@yahoo.com

'Department of Clinical Nutrition \& Dietetics, Faculty of Nutrition Sciences and Food Technology, National Nutrition and Food Technology Research Institute, Shahid Beheshti University of Medical Sciences, Tehran, Iran Full list of author information is available at the end of the article
}

C C The Author(s). 2021 Open Access This article is licensed under a Creative Commons Attribution 4.0 International License, which permits use, sharing, adaptation, distribution and reproduction in any medium or format, as long as you give appropriate credit to the original author(s) and the source, provide a link to the Creative Commons licence, and indicate if changes were made. The images or other third party material in this article are included in the article's Creative Commons licence, unless indicated otherwise in a credit line to the material. If material is not included in the article's Creative Commons licence and your intended use is not permitted by statutory regulation or exceeds the permitted use, you will need to obtain permission directly from the copyright holder. To view a copy of this licence, visit http://creativecommons.org/licenses/by/4.0/ The Creative Commons Public Domain Dedication waiver (http://creativecommons.org/publicdomain/zero/1.0/) applies to the data made available in this article, unless otherwise stated in a credit line to the data. 
(Continued from previous page)

Results: Neither chlorogenic acid nor caffeine was superior to placebo in attenuation of the hepatic fat and stiffness and other hepatic outcomes in patients with diabetes and NAFLD. Except for the lower level of total cholesterol in caffeine group $(p=0.04)$, and higher level of insulin in chlorogenic acid plus caffeine group ( $p=0.01)$ compared with placebo, there were no significant differences among the treatment groups.

Conclusion: These findings do not recommend caffeine and/or chlorogenic acid to treat NAFLD in type 2 diabetes patients.

Trial registration: IRCT201707024010N21. Registered 14 September 2017.

Keywords: Coffee, Caffeine, chlorogenic acid, Type 2 diabetes, Non-alcoholic fatty liver disease, Clinical trial

\section{Introduction}

Patients with type 2 diabetes have a risk of developing serious liver related complications including nonalcoholic fatty liver disease (NAFLD), hepatic cirrhosis, and hepatocellular carcinoma that is two to four times higher than those without diabetes [1].

Observational studies in different populations have suggested that coffee consumption is associated with a reduced risk of NAFLD [2,3], liver fibrosis [4], type 2 diabetes [5], and hepatocellular carcinoma [6]. It is well established that coffee is a mixture of various chemical compounds; among the identified constituents, caffeine, chlorogenic acids, and two diterpenes (cafestol and kahweol) are the main compounds [7]. Caffeine is thought to prevent or reverse hepatic fibrosis by several mechanisms including acting as an $\mathrm{A} 2 \mathrm{~A}$ receptor antagonist which affect the activation of hepatic stellate cells [8]. Recent meta -analysis suggests that this association may be explained by caffeine and not by the decaffeinated coffee. Caffeine is thought to inhibit the proliferation of hepatocellular carcinoma cell [9]. Data from the National Health and Nutrition Examination Surveys (NHANES) that collected between 2001 and 2008 indicated caffeine intake as one of the five independent predictors of NAFLD, suggesting that caffeine may have a potential protective effect [10]. However, a meta- analysis has shown that regular coffee caffeine consumption, but not total caffeine intake, is associated with a lower levels of fibrosis severity [7]. This finding might suggest other ingredients in regular coffee, rather than caffeine, could be responsible for its antifibrotic effects [11]. Chlorogenic acid (a major polyphenol in coffee) plays an important role on modulating glucose intolerance and hyperlipidemia [12], might also play an important role in hepatoprotection. Diterpenes are found in coffee as esters of fatty acid and are known to have a cholesterol raising properties [13]. It is remained unknown what bioactive component in coffee contributes its part more significantly for the reported effects [14].

Although recent meta-analysis of observational studies have shown the beneficial association of coffee consumption with risk reduction of NAFLD and liver fibrosis [15], observational studies can only show association, but cannot provide evidence of causal relationship. The relationship between coffee consumption and liver fibrosis might be due to other factors in frequent coffee drinkers behavior. The hepatoprotective benefits of coffee have not been studied in randomized controlled trials. So, it could not be included in evidence based treatment recommendations. Thus, clinical trials are necessary to find a possible cause-effect relationship, while finding the main effective components of coffee, and their mechanism of action. Therefore, we performed this randomized clinical trial to assess the efficacy and safety of two main components of coffee including caffeine and/or chlorogenic acid at a dose of $200 \mathrm{mg}$ daily in patients who had type 2 diabetes accompanied with NAFLD.

\section{Methods}

Enrollment occurred at two clinics of diabetes in Endocrinology and Metabolism Research Center (Tehran University of Medical Sciences, Tehran, Iran), from September 29, 2017 through March 19, 2018 and the final date of follow up was October 10, 2018. The protocol (available at nimad.ac.ir) was approved by the $\mathrm{Na}$ tional Institute for Medical Research Developments Ethics Committee (IR.NIMAD.REC.1396.028), and all participants provided written informed consent. Capsules containing the caffeine and/ or chlorogenic acid and matching placeboes were provided by Arjuna Natural Extracts, India.

\section{Study population}

Men and women aged 30-53 years with type 2 diabetes (based on 1999 World Health Organization (WHO) criteria) and NAFLD (controlled attenuation parameter (CAP) score of 260 or more) were eligible for enrollment. Exclusion criteria were current use of insulin, current pregnancy or breastfeeding, alcohol intake as defined by an average daily consumption of alcohol $>20 \mathrm{~g} /$ day within the previous year, evidence of other forms of liver diseases (such as viral hepatitis, autoimmune 
hepatitis, etc.), evidence of cirrhosis (according to transient elastography (TE) exam and biochemical profile), current supplementation with vitamin $\mathrm{E}$ and other antioxidants, treatment with Milk thistle (Silybummarianum) (to reduce the effects of confounding factors related to treatment strategies) in the past 6 month or during the study, a history of thyroid, kidney, and mental disease, heart failure, and cancer. Subjects were asked to bring their current medications at the time of screening, and they were checked by the investigator.

\section{Study procedures}

We identified potential patients treated with stable dose of oral anti-hyperglycemic agents and recruited them to fill out a screening questionnaire. Those who answered the questionnaire indicating that they are willing and eligible to participate in the trial, entered in the diagnosis for NAFLD phase, during which, we invited them to undergo abdominal ultrasonography. Patients who had hepatic steatosis of grade 2 or more were examined by TE. Patients who remained eligible (a CAP score level of at least $260 \mathrm{~dB} / \mathrm{m}$ but Liver stiffness measurements (LSM) levels less than $14 \mathrm{kPa}$ ) were randomly assigned by a double-blind fashion using random blocked randomization in a 1:1:1:1 ratio to receive 2 capsules containing either $200 \mathrm{mg}$ of chlorogenic acid plus 200 $\mathrm{mg}$ of caffeine (about 2 cups of coffee), or $200 \mathrm{mg}$ caffeine plus placebo (starch), or $200 \mathrm{mg}$ chlorogenic acid plus placebo, or two matching placebo capsules daily for 6 months. All of investigators involved in this study were blinded to the treatments groups until final analysis. Study visits were conducted at baseline and at $6,12,18$, 24 weeks after initiation of the trial. At each visit, information was gathered regarding adverse events and adherence to study. At the baseline and after 24 weeks of follow up, we collected blood samples, along with anthropometric, blood pressure, and non-invasive markers of hepatic steatosis and fibrosis measurements.

\section{Outcomes}

LSM expressed in kilopascals $(\mathrm{kPa})$ and CAP score expressed in $\mathrm{dB} / \mathrm{m}$ were assessed using FibroScan (EchoSense, Paris, France) in participants fasted for at least $3 \mathrm{~h}$ according to the manufacturer's guideline.

Nutritionist IV software (First Data Bank, The Hearst Corporation, San Bruno, CA) was used for the analysis of $24 \mathrm{~h}$ dietary recalls (5 weekdays and 2 weekend). In addition, physical activity was estimated using the international physical activity questionnaire (IPAQ) by multiplying MET (Metabolic Equivalent of Task) and time spent in each activity [16]. To assess potential changes in daily food consumption and physical activity during the 6-month intervention, participants kept a 7-day food record and a record of their physical activity at baseline and at month 6 of the intervention. All participants were asked to maintain their usual dietary intake and habitual physical activity for the duration of trial.

Resting energy expenditure was measured after $12 \mathrm{~h}$ fasting, using Fitmate Calorimeter (Cosmed, Rome, Italy), while the patients were lied down. Body composition was assessed using body impedance analyzer (BIA) (Tanita, Ilinois, USA).

Blood samplings were performed early in the morning after an overnight fast of 10-12 h. The blood collection from participants, blood withdrawal and blood analyses were done by trained and experienced laboratory technicians in laboratory of Endocrinology and Metabolism Research Institute under suitable conditions. Serum liver enzymes (alanine transaminase (ALT), aspartate transaminase (AST), and gamma-glutamyl transferase (GGT)), total cholesterol (TC), high-density lipoprotein cholesterol (HDL), triglycerides (TG), creatinine (Cr) and high sensitive- $C$ reactive protein (hs-CRP) measured by using ELISA kit (Roche, Germany). Lowdensity lipoprotein cholesterol (LDL) values were calculated using the Friedewald formula. Hemoglobin A1c (HbA1c) levels were assessed by a high performance liquid chromatography analyzer (Tosoh, Tokyo, Japan). Fasting blood glucose levels were measured using the glucose oxidase method on an auto analyzer (Cobas c 311, Roche Dignostics, Risch-Rotkreuz, Switzerland). Serum insulin, C-peptide and thyroid stimulating hormone (TSH) levels were measured by using ELISA kit (Monobind Inc. Lake Forest, California, USA). Homeostasis model assessment-estimated insulin resistance (HOMA-IR) score was used to determine the degree of insulin resistance using the following formula: HOMAIR = Fasting insulin $(\mu \mathrm{U} / \mathrm{mL}) \times$ Fasting glucose $(\mathrm{mmol} /$ L)/22.5. Serum concentrations of total antioxidant capacity (TAC), adiponectin, and cytokeratin-18 (CK-18) were measured using ELISA method (ZellBio $\mathrm{GmbH}$, Ulm, Germany). Serum tumor necrosis factor (TNF)- $\alpha$ was measured by ELISA (Diaclone, France). Nuclear factor $\mathrm{k}-\mathrm{B}$ (NF-kB) levels was assessed in peripheral blood mononuclear cells (PBMCs) nuclear extracts using an ELISA kit (ZellBio GmbH, Ulm, Germany) according to the manufacturer's instructions.

\section{Statistical analysis}

The sample size was calculated for the CAP score, which was based on detection of a 10 unit $(\mathrm{dB} / \mathrm{m})$ difference in the mean CAP score with a power of $80 \%(\beta=20 \%)$, yielding a sample size of 21 for each group. Given the probability of samples loss, twenty five patients in each group were considered [17].

All statistical analyses were performed using SPSS version 16.0 (SPSS Inc., Chicago, IL, USA) on an intentionto-treat basis. Quantitative variables are presented as the 
mean $\pm \mathrm{SD}$. One-way ANOVA were used to examine the consistency of study groups. The Kolmogorov-Smirnov test was used to determine normality of distribution of the examined variables. Non-normally distributed variables were $\log$ transformed. Repeated-measures of ANOVA were used after intervention for assessment of differences between the treatment groups with adjusting for baseline insulin and the baseline value of the outcome being analyzed. Tukey significance difference test was used for post hoc multiple comparisons. Enumeration data were analyzed by the Chi-squared test. Twotailed $P<0.05$ was considered significant.

\section{Results}

From September 2017 through March 2018, a total of 101 patients underwent randomization to receive placebo $(n=23)$, caffeine plus chlorogenic acid $(n=27)$, chlorogenic acid alone $(n=25)$, or caffeine alone $(n=$ 26); however, we excluded one patient from caffeine alone group who had a diagnosis of prediabetes. Totally, a hundred participants comprised the intention- to- treat population, of whom 84 (84\%) completed the study (Fig. 1). There were no meaningful imbalance in demographic characteristics or use of ongoing medications (Table 1), or dietary intake (Table 2), clinical and laboratory data with the exception of insulin levels $(p=0.009)$ (data not shown). The mean age of all patients was $44.57 \pm 5.65$ years, with a mean diabetes duration of $4.57 \pm 4.23$ years and the majority of them were male (68\%). There was no significant difference in dietary intakes among the groups before and after the intervention.

Table 3 shows the differences in hepatic indicators, metabolic profile, inflammatory and total antioxidant levels among treatment groups at the end of study adjusted for the baseline values and insulin levels. CAP scores and LSM were not significantly different among the groups. Similarly, no significant differences between

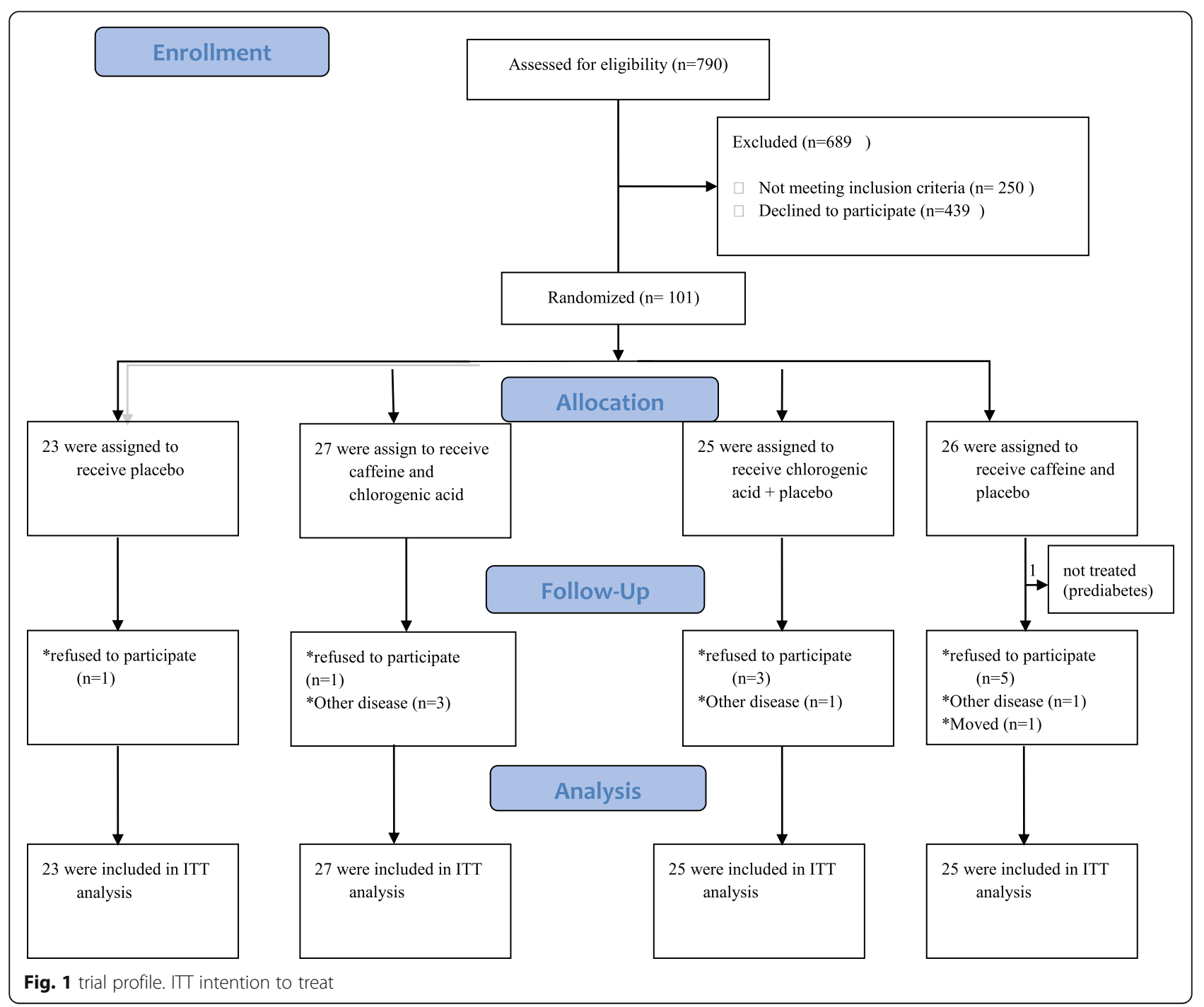


Table 1 Demographic characteristics, anthropometrics and use of ongoing medications of study population according to group of treatment at baseline

\begin{tabular}{|c|c|c|c|c|c|}
\hline Characteristic & $\begin{array}{l}\text { Placebo } \\
(n=23)\end{array}$ & $\begin{array}{l}\text { Cholrogenic acid+ caffeine } \\
(n=27)\end{array}$ & $\begin{array}{l}\text { Cholrogenic acid } \\
(n=25)\end{array}$ & $\begin{array}{l}\text { Caffeine } \\
(n=25)\end{array}$ & Pvalue \\
\hline Age (yr) & $44.17 \pm 4.98$ & $43.59 \pm 5.51$ & $44.88 \pm 6.91$ & $45.68 \pm 5.07$ & $0.58^{*}$ \\
\hline Male sex, No (\%) & $15(65.2)$ & $17(63)$ & 19(76) & $17(68)$ & $0.76^{*}$ \\
\hline Known duration of diabetes (yr) & $4.05 \pm 4.49$ & $3.84 \pm 3.65$ & $4.37 \pm 3.25$ & $5.97 \pm 5.27$ & $0.22^{¥}$ \\
\hline Number of current smokers (\%) & $4(17.4)$ & $7(25.9)$ & $3(12)$ & $4(16)$ & $0.6^{*}$ \\
\hline Height (cm) & $170.91 \pm 9.83$ & $170.13 \pm 9.74$ & $172.48 \pm 8.04$ & $170.32 \pm 9.76$ & $0.8^{¥}$ \\
\hline BMI $\left(\mathrm{kg} / \mathrm{m}^{2}\right)$ & $30.6 \pm 3.1$ & $31.26 \pm 4.76$ & $30.84 \pm 3.28$ & $29.59 \pm 3.4$ & $0.45^{¥}$ \\
\hline Fat mass (kg) & $27.23 \pm 7.16$ & $28.9 \pm 10.31$ & $26.47 \pm 6.94$ & $24.74 \pm 8.27$ & $0.35^{*}$ \\
\hline FFM (kg) & $62.1 \pm 11.29$ & $61.48 \pm 12.33$ & $64.54 \pm 10.95$ & $60.58 \pm 9.59$ & $0.67^{*}$ \\
\hline Waist circumferences (cm) & $107.48 \pm 9.12$ & $108.91 \pm 10.9$ & $110.26 \pm 9.5$ & $104.6 \pm 6.87$ & $0.17^{¥}$ \\
\hline REE (kcal) & $1833.37 \pm 395.42$ & $1859.59 \pm 381.41$ & $1865 \pm 339.25$ & $1750.09 \pm 314.37$ & $0.68^{*}$ \\
\hline Physical activity (METs h/day) & $30.52 \pm 5.19$ & $30.54 \pm 4.08$ & $32.18 \pm 4.39$ & $31.75 \pm 3.94$ & $0.43^{¥}$ \\
\hline \multicolumn{6}{|l|}{ Medications, n(\%) } \\
\hline Metformin & $20(87)$ & $23(85)$ & 23(92) & $21(84)$ & $0.84^{*}$ \\
\hline DPP4 Inhibitors & $6(26.1)$ & $6(22.2)$ & $4(16)$ & $11(44)$ & $0.14^{*}$ \\
\hline Sulfonamide & $5(21.7)$ & $9(33.3)$ & $10(40)$ & $9(36)$ & $0.57^{*}$ \\
\hline Beta blocker+ABRS & $6(26.1)$ & $6(22.2)$ & $3(12)$ & $4(16)$ & $0.59^{*}$ \\
\hline Statin & $6(26)$ & $10(37)$ & $10(40)$ & $11(44)$ & $0.61^{*}$ \\
\hline Other lipid lowering gents & 0 & $3(11.1)$ & $2(8)$ & $1(4)$ & $0.38^{*}$ \\
\hline Aspirin & $3(13)$ & $2(7.4)$ & 2(8) & $4(16)$ & $0.72^{*}$ \\
\hline
\end{tabular}

¥ One-way ANOVA

${ }^{*}$ chi- square

Two-tailed $P<0.05$ was considered significant

Each value represents mean \pm SD except for gender, smoking, ongoing use of medication $n(\%)$. BMI body mass index, FFM fat free mass, REE resting energy expenditure, METs Metabolic Equivalents, DPP4 Dipeptidyl Peptidase 4, ARBS Angiotensin II receptor blockers

Table 2 Energy and nutrients intake among treatment groups at baseline and after 6 months

\begin{tabular}{|c|c|c|c|c|c|}
\hline Characteristic & $\begin{array}{l}\text { Placebo } \\
(n=23)\end{array}$ & $\begin{array}{l}\text { Cholrogenic acid+ caffeine } \\
(n=27)\end{array}$ & $\begin{array}{l}\text { Cholrogenic acid } \\
(n=25)\end{array}$ & $\begin{array}{l}\text { Caffeine } \\
(n=25)\end{array}$ & Pvalue \\
\hline \multicolumn{6}{|c|}{ Energy intake (kcal) } \\
\hline Baseline & $2536.16 \pm 403.88$ & $2603.12 \pm 595.55$ & $2626 \pm 485.68$ & $2855.62 \pm 445.29$ & $0.32^{*}$ \\
\hline 6 months & $2313.42 \pm 441.25$ & $2459.41 \pm 432.01$ & $2412.63 \pm 402.49$ & $2385.50 \pm 495.96$ & $0.08^{*}$ \\
\hline \multicolumn{6}{|l|}{ Protein (g) } \\
\hline Baseline & $79 \pm 23.3$ & $85.61 \pm 31.94$ & $80.35 \pm 28.26$ & $93.46 \pm 6.56$ & $0.5^{*}$ \\
\hline 6 months & $70.24 \pm 20.47$ & $83.89 \pm 20.65$ & $75.41 \pm 18.99$ & $82.56 \pm 30$ & $0.6^{*}$ \\
\hline \multicolumn{6}{|l|}{ Fat(g) } \\
\hline Baseline & $120.14 \pm 18.23$ & $121.38 \pm 28.22$ & $105.65 \pm 23.48$ & $124.9 \pm 18.04$ & $0.13^{*}$ \\
\hline 6 months & $108.32 \pm 27.22$ & $98.77 \pm 34.69$ & $100.99 \pm 17.69$ & $107.81 \pm 25.41$ & $0.36^{*}$ \\
\hline \multicolumn{6}{|l|}{ Carbohydrate (g) } \\
\hline Baseline & $293.9 \pm 71.37$ & $302.16 \pm 89.07$ & $351.04 \pm 53.98$ & $350.55 \pm 85.39$ & $0.1^{*}$ \\
\hline 6 months & $237.22 \pm 66.01$ & $320.1 \pm 95.12$ & $309.87 \pm 70.03$ & $283.15 \pm 73.11$ & $0.15^{*}$ \\
\hline \multicolumn{6}{|l|}{ Caffeine $(\mathrm{mg})^{* *}$} \\
\hline Baseline & $79.75 \pm 42.94$ & $97.11 \pm 51.08$ & $136 . .55 \pm 105.74$ & $130.68 \pm 82.77$ & $0.16^{*}$ \\
\hline 6 months & $131.78 \pm 98.35$ & $117.28 \pm 82.02$ & $110.32 \pm 90.87$ & $130.51 \pm 125.67$ & $0.37^{*}$ \\
\hline
\end{tabular}

Data are mean \pm SD

¥ One-way ANOVA

*Time X Treatment interaction according to two-way repeated measures ANOVA

**The amount of caffeine consumed in foods and beverages

Two-tailed $\mathrm{P}<0.05$ was considered significant 
Table 3 Changed in hepatic indicators, metabolic profile, inflammatory and total antioxidant levels vs. placebo

\begin{tabular}{|c|c|c|c|}
\hline \multirow[t]{2}{*}{ Characteristic } & \multicolumn{3}{|l|}{ Adjusted treatment differences $(95 \% \mathrm{Cl} ; \mathrm{p})$} \\
\hline & Chlorogenic acid+ caffeine vs placebo & Chlorogenic acid vs placebo & Caffeine vs placebo \\
\hline Fibro score (KPa) & $0.43(-0.46$ to $1.32 ; p=0.3)$ & $0.66(-0.21$ to $1.53 ; P=0.09)$ & $0.24(-0.62$ to $1.12 ; P=0.57)$ \\
\hline CAP score $(\mathrm{dB} / \mathrm{m})$ & $12.06(-7.3$ to $31.46 ; P=0.26)$ & $13.07(-5.6$ to $32.13 ; P=0.2)$ & $-7.45(-26.48$ to $11.57 ; P=0.31)$ \\
\hline CK-18 fragments (U/L) & $-0.00(-0.22$ to $0.21 ; P=0.42)$ & $0.04(-0.16$ to $0.25 ; P=0.85)$ & $-0.13(-0.33$ to $0.08 ; P=0.17)$ \\
\hline $\mathrm{AST}(\mathrm{U} / \mathrm{L})$ & $2.11(-2.7$ to $6.97 ; P=0.37)$ & $3.07(-1.71$ to $7.85 ; P=0.13)$ & $-0.43(-5.19$ to $4.34 ; P=0.87)$ \\
\hline $\operatorname{ALT}(U / L)$ & $1.9(-2.45$ to $6.23 ; P=0.62)$ & $2.4(-1.8$ to $6.7 ; P=0.17)$ & $-0.12(-4.4$ to $4.13 ; P=0.79)$ \\
\hline GGT(U/L) & $0.78(-16.68$ to $15.13 ; P=0.94)$ & $3.48(-12.13$ to $19.1 ; P=0.41)$ & $8.55(-7.05$ to $24.16 ; P=0.35)$ \\
\hline Fasting glucose (mg/dl) & $-1.76(-30.08$ to $26.46 ; P=0.97)$ & $4.9(-22.9$ to $32.7 ; P=0.74)$ & $-2.99(-30.77$ to $24.78 ; P=0.86)$ \\
\hline $\mathrm{HbA1C}(\%)$ & $-0.25(-1.21$ to $0.71 ; P=0.66)$ & $0.04(-0.9$ to $1 ; P=0.98)$ & $-0.55(-1.5$ to $0.39 ; P=0.18)$ \\
\hline Insulin (ulU/ml) & $3.3(-1.3$ to $6.7 ; P=0.01)$ & $0.2(-3.28$ to $3.7 ; P=0.67)$ & $-0.16(-3.66$ to $3.32 ; P=0.71)$ \\
\hline C-peptide (ng/ml) & $0.04(-0.27$ to $0.36 ; P=0.96)$ & $-0.03(-0.35$ to $0.28 ; P=0.84)$ & $-0.06(-0.37$ to $0.26 ; P=0.51)$ \\
\hline HOMA-IR score & $0.4(-0.62$ to $1.42 ; P=0.69)$ & $0.01(-0.99$ to $1.01 ; P=0.78)$ & $-0.01(-1.02$ to $0.99 ; P=0.91)$ \\
\hline $\mathrm{TG}(\mathrm{mg} / \mathrm{dl})$ & $-10.56(-140.54$ to $119.42 ; P=0.89)$ & $46.17(-81.45$ to $173.79 ; P=0.65)$ & $-62.99(-190.50$ to $64.53 ; P=0.17)$ \\
\hline Cholesterol (mg/dl) & $-9.43(-29.7$ to $10.83 ; P=0.5)$ & $-5.07(-24.97$ to $14.82 ; P=0.7)$ & $-20.66(-40.55$ to $-0.78 ; P=0.04)$ \\
\hline $\mathrm{HDL}(\mathrm{mg} / \mathrm{dl})$ & $-0.94(-5.83$ to $3.95 ; P=0.79)$ & $0.27(-4.5$ to $5.07 ; P=0.89)$ & $1.62(-3.18$ to $6.41 ; P=0.52)$ \\
\hline LDL (mg/dl) & $-5.77(-21.3$ to $9.76 ; P=0.49)$ & $-5.58(20.98$ to $9.81 ; P=0.48)$ & $-11.93(-27.16$ to $3.3 ; P=0.12)$ \\
\hline hs-CRP (mg/dl) & $0.73(-2.61$ to $4.08 ; P=0.6)$ & $-1.64(-4.93$ to $1.64 ; P=0.33)$ & $0.02(-3.25$ to $3.31 ; P=0.99)$ \\
\hline TNF-a (pg/ml) & $0.5(-8.27$ to $9.2 ; P=0.83)$ & $-4.96(-13.33$ to $3.4 ; P=0.24)$ & $-1.59(-10.02$ to $6.84 ; P=0.96)$ \\
\hline NF-KB (ng/mg protein) & $-0.16(-1.08$ to $0.77 ; P=0.68)$ & $0.23(-0.67$ to $1.14 ; P=0.9)$ & $-0.23(-0.93$ to $0.88 ; P=0.8)$ \\
\hline Adiponectin (mg/L) & $0.29(-1.82$ to $2.4 ; P=0.92)$ & $0.07(-1.95$ to $2.1 ; P=0.86)$ & $-2.43(-4.47$ to $-0.4 ; P=0.06)$ \\
\hline $\mathrm{TAC}(\mathrm{mmol} / \mathrm{L})$ & $0.00(-0.04$ to $0.05 ; P=0.69)$ & $-0.00(-0.05$ to $0.04 ; P=0.92)$ & $0.00(-0.04$ to $0.04 ; P=0.79)$ \\
\hline $\mathrm{Cr}(\mathrm{mg} / \mathrm{dl})$ & $-0.00(-0.1$ to $0.09 ; P=0.84)$ & $0.02(-0.07$ to $0.11 ; P=0.63)$ & $0.07(-0.02$ to $0.17 ; P=0.12)$ \\
\hline TSH (mIU/L) & $-0.11(-0.9$ to $0.66 ; P=0.32)$ & $0.24(-0.52$ to $1.01 ; P=0.85)$ & $0.06(-0.71$ to $0.82 ; P=0.75)$ \\
\hline Weight (kg) & $0.63(-6.33$ to $7.59 ; p=0.51)$ & $2.36(-4.73$ to $9.45 ; p=0.63)$ & $-3.39(-10.49$ to3.7: $p=0.38)$ \\
\hline Systolic blood pressure (mmHg) & $0.26(-6.93$ to $7.45 ; P=0.84)$ & $1.02(-6.04$ to $8.08 ; P=0.68)$ & $-1.41(-8.47$ to $5.65 ; P=0.83)$ \\
\hline Diastolic blood pressure $(\mathrm{mmHg})$ & $-1.69(-6.83$ to $3.46 ; P=0.59)$ & $-1.27(-6.32$ to $3.78 ; P=0.77)$ & $-1.2(-6.25$ to $3.84 ; P=0.83)$ \\
\hline
\end{tabular}

${ }^{2}$ Tukey significance difference test was used for post hoc multiple comparisons. Two-tailed $P<0.05$ was considered significant

Data not conforming to a normal distribution were log-transformed prior to parametric analysis. CAP -Score controlled attenuation parameter Score, CK-18 cytokeratin-18, AST aspartate aminotransferase, ALT alanine aminotransferase, GGT gamma-glutamyltransferase, HbA1c hemoglobinA1c, HOMA-IR homeostasis model assessment insulin resistance, TG Triglyceride, HDL high-density lipoprotein, LDL low-density lipoprotein, hs-CRP high-Sensitivity C-Reactive Protein, TNF-a tumor necrosis factor- $a$, NF-KB nuclear factor kappa B, TAC total antioxidant capacity, Cr creatinine, TSH thyroid stimulating hormone

groups were evident in other hepatic indicators including AST, ALT, GGT, Ck-18 levels $(p>0.05)$. Except for increase in fasting insulin (mean differences $3.3 \mu \mathrm{IU} / \mathrm{ml}$ [95\%CI, -1.3 to 6.7]; $p=0.01$ ), in chlorogenic acid plus caffeine compared with placebo, none of the other supplementations were significantly associated with changes in fasting glucose, HOMA-IR, HBA1C or C-peptide compared with placebo. Supplementation with caffeine significantly decreased cholesterol levels as compared with placebo (mean differences $-20.66 \mathrm{mg} / \mathrm{dl}$ [ $95 \% \mathrm{CI}$, 40.55 to -0.78$] ; p=0.04$ ), whereas LDL- cholesterol, HDL- cholesterol, and TG were not affected by any other groups of treatments. No other significant differences among the four groups were found in those treated with caffeine or/ and chlorogenic acid compared with placebo regarding all other biomarkers (NF-KB,
TNF- $\alpha$, hs-CRP, TAC, adiponectin, $\mathrm{Cr}$ and TSH). Moreover, systolic and diastolic blood pressure changes did not differ significantly among the groups. No side effects were reported during the study period.

\section{Discussion}

This is the first randomized, double-blind, placebocontrolled trial of chlorogenic acid and/ or caffeine supplementation performed in type 2 diabetes patients with NAFLD. This trial showed that an intervention based on main components of coffee has no significant effect on hepatic fat content and fibrosis in type 2 diabetic patients affected by NAFLD. Six months supplementation with caffeine and/or chlorogenic acid did not improve either hepatic enzymes (ALT, AST, and GGT) or the serum levels of CK-18 as a specific biomarker for liver 
injury. As we specifically aimed to investigate the effects of coffee main constitutes on NAFLD in patients with diabetes, we also tested the hypothesis of an involvement of chlorogenic acid and/ or caffeine in modulating insulin resistance (HOMA-IR), metabolic and glycemic control (fasting glucose, HBA1C) in these subjects. Our results have shown that except for increase in insulin in chlorogenic acid plus caffeine group, and decrease in cholesterol in caffeine group, none of these parameters significantly changed after 6 months intervention in comparison with the placebo group. Likewise, the supplementation did not induce any specific effect on thyroid and renal function as measured by TSH and $\mathrm{Cr}$ levels, respectively. Similarly, neither inflammatory indictors nor antioxidant capacity changes were significantly different between active treated groups and placebo after 6 months.

Decaffeinated and regular coffee consumption have been investigated in prevention and treatment of NAFL $D$ with varying results in many large observational studies; most of which reported that coffee has beneficial impact on liver; however rarely, it was the main target of interest or studied in clinical trials $[3,18]$. In pre -clinical studies, caffeine seems to be able to exert inhibitory effects on fibrogenesis and block the progression of liver steatosis through interruption in transforming growth factor- $\beta$ (TGF- $\beta$ ) signaling, inhibition of stellate cells and by its antioxidant and anti-inflammatory properties [19]. However, contrary to these hypotheses, we observed that caffeine and / or chlorogenic acid did not significantly affect liver outcomes after 6 months supplementation. Coffee contains other substances (e.g. melanoidins, niacin, diterphenoic alcohols) in addition to caffeine and chlorogenic acid that could exert a protective effect on a range of liver diseases $[18,20]$. In the present study, the amount of caffeine or chlorogenic acid ingested by the subjects was $200 \mathrm{mg} /$ day, which equates to the amount of caffeine found in 2 cups of coffee, which might not be enough to show any effect. Observational studies showed that drinking more than 3 cups coffee per day is protective against fatty liver $[18,21]$. Since increase in blood pressure with regular coffee or caffeine intake have been observed [22], we were not ensured that most of our patients would easily tolerate upper doses of coffee (above $200 \mathrm{mg} / \mathrm{d}$ ). However, this study did not provide support for the adverse relationship between coffee and blood pressure and even led to non-significant $1.2-1.69 \mathrm{mmHg}$ decrease in diastolic blood pressure. A meta- analysis of clinical trials and prospective cohort studies' results are in consistent with our study, suggesting no evidence of any relationship between coffee consumption and hypertension [23]. Zhang et al. described an inverse J-shaped association between intake of this beverage and hypertension risk; so that one to three cups coffee/day intake compared with less than one cup/day could increase the risk, while protective effects were observed at higher intakes [24]. Another meta-analyses concluded a beneficial effect of coffee on blood pressure $[25,26]$. It is important to note that sex, race, and lifestyle factors (e.g., smoking) have been considered as factors involved in the different shape of relationship between coffee use and blood pressure [22]. In addition, inter -individual differences mainly due to the variation in caffeine metabolism related genes including CYP1A2 (slow caffeine metabolizers vs. rapid metabolizers) may partially explain conflicting results [24]. As we aimed specifically to assess the efficacy of chlorogenic acid and/ or caffeine supplementation on liver steatosis in type 2 diabetes patients, our sample consisted of patients attending to our diabetes outpatients' center for routine diabetes care. So, this setting led to recruitment of cases with mild degree of NAFLD rather than those with advanced liver disease referring to hepatology clinics. The results from Bambha et al. study showed that in those with less insulin resistance, high coffee consumption was inversely associated with advanced fibrosis, as compared with those with greater insulin resistance. So, it could speculate less benefit of coffee consumption on liver fibrosis treatment in patients with diabetes who have insulin resistance [14]. Indeed, we cannot rule out the effect of coffee components in NAFLD patients with greater fibrosis or patients with less insulin resistance or non-diabetic patients. The lack of significant effects of coffee on liver outcomes are consistent with the non-significant effects of coffee on antioxidant and inflammatory and anti-inflammatory biomarkers. Similar to the results from previous trials, we did not find any effects of coffee on hs-CRP [27, 28], and TNF- $\alpha$ [29]. In this study, no change in NF-KB activity in PBMCs was also observed. The lack of antioxidant and anti-inflammatory responses in the present study could partly mediate the non-significant effect of supplements on liver after caffeine and / or chlorogenic acid ingestion. Although few studies in the past have attempted to assess the effect of caffeine on inflammatory biomarkers, the findings remained unclear mainly as a result of different study designs and outcomes [30]. A recent systematic review has concluded that coffee consumption was either neutrally or inversely associated with serum concentrations of inflammatory markers [30].

In a meta-analysis on cross sectional studies between 2008 and 2017, Zhang et al. showed that coffee consumption is efficient to increase adiponectin levels only in females [31]. This finding could be explained by the difference in number of fat cells between men and women [31]. In addition, intake of $4 \leq$ cups of coffee in women with and without diabetes was also associated with $20 \%$ increase in adiponectin levels [32]. In contrast, 
Wedick et al. found increase in adiponectin levels in both genders after 8 weeks of supplementation with 5 cups of coffee/d [27]. However, in a cross sectional study on multi-ethnic Asian population, there was no relationship between coffee drinking and adiponectin concentration [33]. Our data are corroborated by the finding of this study which did not observe differences in levels of adiponectin even after including both genders. Green coffee bean extract supplementation in NAFLD patients induced significant increase in TAC and decrease in hsCRP, but not in TNF- $\alpha$ [34]. Consistent with these results, a study of healthy adults who drank coffee which contained medium and high chlorogenic acid reported positive change in antioxidant capacity [35]. Our finding, however, indicate that chlorogenic acid has no major effect on antioxidant capacity in diabetic patients.

Evidence concerning the effect of regular and decaffeinated coffee on insulin sensitivity is controversial, especially as data provided by short term trials and observational studies have reached discordant results and conclusions [12]. Since 2002, many but not all observational studies have found potential beneficial effect of both caffeinated and decaffeinated coffee consumption against the incidence of developing type 2 diabetes by $30-60 \%$ in a diverse population $[12,36]$. In addition, previous experimental studies have shown that chlorogenic acid is responsible for hypoglycemic effects of coffee. This result can be explained by inhibition of glucose absorption and at the cellular level, activating AMPactivated protein kinase (AMPK) [12]. However, the observational and experimental studies are not confirmed by all human short term clinical trials [37, 38].

No significant effect was found on glucose metabolism in our study, similar to previous report that evaluated the effect of coffee on this variable in type 2 diabetes [28]. We showed that chlorogenic acid may act synergistically with caffeine to increase insulin levels. This finding is consistent with a short term trial of healthy volunteers in which caffeine administrated in capsulated form ( $400 \mathrm{mg}$ in two capsules or $870 \mathrm{mg}$ in six capsules) or coffee drink (52 g coffee grounds) or roasted coffee $(300 \mathrm{ml})$ [37-39]. From the result of a recent meta -analysis conducted in healthy subjects, it has been found that acute caffeine ingestion reduces insulin sensitivity [40]. However, Da Silva et al. suggested that caffeine consumption is associated with increase in insulin clearance [41]. The mechanism of action could be explained by the effects of caffein on increasing epinephrine release and free fatty acids levels or activation of adenosine receptor [27, 38]. However, tolerance to some effects of caffeine may be developed over time [27]. Moreover, caffeine stimulates insulin secretion primarily by activation of ryanodine receptors in pancreatic $\beta$-cells in a glucose dose dependent manner, with no effect at low glucose concentrations and stimulating insulin only when glucose concentration is high [42]. This mechanism may explain why caffeine has increased insulin levels in diabetic patients in our long term study.

The association between coffee consumption and serum lipids have been studied extensively, however, yielded inconsistent results [28]. A meta- analysis of clinical trials suggested possible increased serum levels of LDL-C and total cholesterol for unfiltered coffee consumption [43], but this finding was partially driven by the results of English language literature and two important trials [44, 45], while studies published before 1998 were not identified. Additional data from a metaanalysis including only randomized controlled trials involving a total of 1017 subjects provided more information about any potential effects of coffee consumption; it suggested that the serum levels of TC, LDL-C and TG may significantly increase by coffee consumption, but the effect was greater for boiled (unfiltered) coffee [46]. Although studies that used filtered coffee (removed the most of the oils by paper filter) have reported conflicting results, some studies found positive effects (decrease in cholesterol and LDL-C levels [47] and increase in HDLC) [28], while other studies did not find any effects of coffee consumption on components of the lipid profile [48]. The preparation method of coffee can affect the concentrations of lipid altering factors such as cafestol and kahweol and caffeine [49]; however, the effects of caffeine on serum cholesterol remained unclear [46]. In one observational study, investigators have suggested that coffee consumption in a dose -dependent manner is associated with lipid-raising effect, regardless of its caffeine content [50].

Although a case-control study found an unfavorable association between caffeine consumption and cholesterol [51], one of the finding of present study was that caffeine intake $(200 \mathrm{mg} / \mathrm{d})$ was associated with about $20 \mathrm{mg} / \mathrm{dl}$ decrease in total cholesterol over 6 months of follow up after adjustment for potential confounders. Increase of fecal lipid extraction has been suggested as a possible mechanism underling the cholesterol -decreasing effect of caffeine [52]. In addition, in observational studies, many people do not consume coffee in isolation, but add sugar (glucose and fructose) and milk which might be responsible for increase in cholesterol levels [46].

To summarize, we did not meet the primary endpoint of showing a reduction in hepatic fat and fibrosis in NAFLD patients undergoing 6 months supplementation. Indeed, it is possible to speculate that either the period of exposure was not enough for modifying the hepatic fat and fibrosis and the biochemical indicators of hepatic involvement in NAFLD, or the link between coffee and NAFLD could be appreciated only in specific 
subpopulation of NAFLD patients. As we enrolled diabetic patients with the number of patients with few patients with advanced fibrosis, the results cannot be generalized to other population and would be different if non diabetic patients or NASH patients with advanced fibrosis were included in the study. Furthermore, we used the dose of $200 \mathrm{mg}$ of each supplement, while some observational studies indicate that higher doses might be effective.

This study has several advantages. This is the first randomized double-blind, placebo-controlled, clinical trial that evaluated the effects of main components of coffee on patients with NAFLD and diabetes. Using the extracted components of coffee provided us an opportunity to roll out the confounding effects of coffee preparation methods, varieties in different coffee components, and variation in the amount of coffee consumption. Since our study population had a background of lower caffeine consumption (i.e. irregular coffee intake or coffee abstinence), this might resulted in more accurate findings on the studied parameters, as a tolerance to the effects of caffeine probably would not have already developed. Furthermore, we asked the patients to take supplements in the morning, which is usual mode of coffee intake. Furthermore, we combined chlorogenic acid and caffeine to determine if there could be increased benefit from adjuvant therapy. In addition, because all participants were newly diagnosed by NAFLD, they were not undertaken any treatment as a part of clinical care, which reduced the effects of confounding factors related to treatment strategies.

\section{Conclusion}

Our study found that six months supplementation with $200 \mathrm{mg} /$ day of two main components of coffee had no significant impact on non-invasive markers of hepatic steatosis and fibrosis, liver biochemistry, inflammatory and metabolic parameters in patients with NAFLD and type 2 diabetes; however, this dosage was completely safe without any reported side-effects. Further trials with higher dosages, longer duration, and different active components of coffee are highly recommended.

\footnotetext{
Abbreviations

NAFLD: Non-alcoholic fatty liver disease; WHO: World Health Organization; CAP: Controlled attenuation parameter; TE: Transient elastography; LSM: Liver stiffness measurements; IPAQ: International physical activity questionnaire; METs: Metabolic Equivalent of Task; BIA: Body impedance analyzer; ALT: Alanine transaminase; AST: Aspartate transaminase; GGT: Gammaglutamyl transferase; TC: Total cholesterol; HDL: High-density lipoprotein cholesterol; TG: Triglycerides; Cr : Creatinine; hs-CRP: High sensitive- C reactive protein; LDL: Low-density lipoprotein cholesterol; HbA1c: Hemoglobin A1c; TSH: Thyroid stimulating hormone; HOMAIR: Homeostasis model assessment-estimated insulin resistance; TAC: Total antioxidant capacity; CK-18: Cytokeratin-18; PBMC: Peripheral blood
}

mononuclear cell; TGF- $\beta$ : Transforming growth factor- $\beta$; AMPK: AMPactivated protein kinase

\section{Acknowledgements}

We are thanks to the patients who participated in the study. We also thank Dr. Maryam Sharafkhah for her consultation in data analysis.

\begin{abstract}
Authors' contributions
A.M. Study conception and design, Acquisition of data, Analysis and interpretation of data, Drafting of manuscript, Critical revision. M.R.M.T. Critical revision. M.S. Acquisition of data. M.Q. Analysis and interpretation of data. SH.M. Study conception and design. H.A. Acquisition of data. H.P. Acquisition of data. A.H. Study conception and design, Critical revision. The author(s) read and approved the final manuscript.
\end{abstract}

\section{Funding}

This study was supported by the National Institute for Medical Research Developments, and by National Nutrition and Food Technology Research Institute, Shahid Beheshti of Medical Sciences, Tehran, Iran.

\section{Availability of data and materials}

Data are available upon reasonable request.

\section{Declarations}

Ethical approval and consent to participate

The protocol (available at nimad.ac.ir) was approved by the National Institute for Medical Research Developments Ethics Committee, and all participants provided written informed consent. All patients were provided written informed consent.

\section{Consent for publication}

Not applicable.

\section{Competing interests}

None of the authors have any conflicts of interest or financial ties to disclose.

\section{Author details}

${ }^{1}$ Department of Clinical Nutrition \& Dietetics, Faculty of Nutrition Sciences and Food Technology, National Nutrition and Food Technology Research Institute, Shahid Beheshti University of Medical Sciences, Tehran, Iran. ${ }^{2}$ Endocrinology and Metabolism Research Center, Endocrinology and Metabolism Clinical Sciences Institute, Tehran University of Medical Sciences, Tehran, Iran. ${ }^{3}$ Radiology Department, Shariati Hospital, Tehran University of Medical Sciences, Tehran, Iran. ${ }^{4}$ Non-communicable Diseases Research Center, Alborz University of Medical Sciences, Karaj, Iran. ${ }^{5}$ Chronic Diseases Research Center, Endocrinology and Metabolism Population Sciences Institute, Endocrinology and Metabolism Research Institute, Tehran University of Medical Sciences, Tehran, Iran. ${ }^{6}$ Liver and Pancreatobiliary Diseases Research Center, Digestive Diseases Research Institute, Tehran University of Medical Sciences, Tehran, Iran. ${ }^{7}$ Diabetes Research Center, Endocrinology and Metabolism Clinical Sciences Institute, Tehran University of Medical Sciences, Tehran, Iran.

Received: 22 July 2020 Accepted: 3 April 2021

Published online: 10 April 2021

\section{References}

1. Mantovani A, Byrne CD, Bonora E, Targher G. Nonalcoholic fatty liver disease and risk of incident type 2 diabetes: a meta-analysis. Diabetes Care. 2018; 41(2):372-82. https://doi.org/10.2337/dc17-1902.

2. Poole R, Kennedy OJ, Roderick P, Fallowfield JA, Hayes PC, Parkes J. Coffee consumption and health: umbrella review of meta-analyses of multiple health outcomes. BMJ (Clinical research ed). 2017;359:j5024.

3. Wijarnpreecha K, Thongprayoon C, Ungprasert P. Coffee consumption and risk of nonalcoholic fatty liver disease: a systematic review and metaanalysis. Eur J Gastroenterol Hepatol. 2017;29(2):e8-e12. https://doi.org/10.1 097/MEG.0000000000000776.

4. Dranoff JA, Feld JJ, Lavoie ÉG, Fausther M. How does coffee prevent liver fibrosis? Biological plausibility for recent epidemiological observations. Hepatology. 2014;60(2):464-7. https://doi.org/10.1002/hep.27032. 
5. Ding M, Bhupathiraju SN, Chen M, van Dam RM, Hu FB. Caffeinated and decaffeinated coffee consumption and risk of type 2 diabetes: a systematic review and a dose-response meta-analysis. Diabetes Care. 2014;37(2):569-86. https://doi.org/10.2337/dc13-1203.

6. Setiawan W, Wilkens LR, Lu SC, Hernandez BY, Le Marchand L, Henderson $B E$. Association of coffee intake with reduced incidence of liver cancer and death from chronic liver disease in the US multiethnic cohort. Gastroenterology. 2015;148(1):118-25. https://doi.org/10.1053/j.gastro.2014.1 0.005 .

7. Shen H, Rodriguez AC, Shiani A, Lipka S, Shahzad G, Kumar A, et al. Association between caffeine consumption and nonalcoholic fatty liver disease: a systemic review and meta-analysis. Ther Adv Gastroenterol. 2016; 9(1):113-20. https://doi.org/10.1177/1756283X15593700.

8. Wang H, Guan W, Yang W, Wang Q, Zhao H, Yang F, et al. Caffeine inhibits the activation of hepatic stellate cells induced by acetaldehyde via adenosine A2A receptor mediated by the CAMP/PKA/SRC/ERK1/2/P38 MAPK signal pathway. PloS one. 2014;9(3).

9. Kennedy OJ, Roderick P, Buchanan R, Fallowfield JA, Hayes PC, Parkes J. Coffee, including caffeinated and decaffeinated coffee, and the risk of hepatocellular carcinoma: a systematic review and dose-response metaanalysis. BMJ Open. 2017;7(5).

10. Birerdinc A, Stepanova M, Pawloski L, Younossi Z. Caffeine is protective in patients with non-alcoholic fatty liver disease. Aliment Pharmacol Ther. 2012;35(1):76-82. https://doi.org/10.1111/j.1365-2036.2011.04916.x.

11. Kennedy OJ, Roderick P, Poole R, Parkes J. Coffee, caffeine and nonalcoholic fatty liver disease? Ther Adv Gastroenterol. 2016;9(3):417-8. https:// doi.org/10.1177/1756283X16636765

12. Reis CE, Dórea JG, da Costa TH. Effects of coffee consumption on glucose metabolism: a systematic review of clinical trials. J Tradit Complement Med. 2018.

13. Costabile A, Sarnsamak K, Hauge-Evans AC. Coffee, type 2 diabetes and pancreatic islet function-a mini-review. J Funct Foods. 2018;45:409-16. https://doi.org/10.1016/j.jff.2018.04.011.

14. Bambha K, Wilson LA, Unalp A, Loomba R, Neuschwander-Tetri BA, Brunt EM, et al. Coffee consumption in NAFLD patients with lower insulin resistance is associated with lower risk of severe fibrosis. Liver Int. 2014; 34(8):1250-8. https://doi.org/10.1111/liv.12379.

15. Hayat U, Siddiqui AA, Okut H, Afroz S, Tasleem S, Haris A. The effect of coffee consumption on the non-alcoholic fatty liver disease and liver fibrosis: a meta-analysis of 11 epidemiological studies. Ann Hepatol. 2020;20: 100254.

16. Craig CL, Marshall AL, Sjöström M, Bauman AE, Booth ML, Ainsworth BE, et al. International physical activity questionnaire: 12-country reliability and validity. Med Sci Sports Exerc. 2003;35(8):1381-95. https://doi.org/10.1249/01. MSS.0000078924.61453.FB.

17. Eslamparast T, Poustchi H, Zamani F, Sharafkhah M, Malekzadeh R, Hekmatdoost A. Synbiotic supplementation in nonalcoholic fatty liver disease: a randomized, double-blind, placebo-controlled pilot study. Am J Clin Nutr. 2014:99(3):535-42. https://doi.org/10.3945/ajcn.113.068890.

18. Chen Y-P, Lu F-B, Hu Y-B, Xu L-M, Zheng M-H, Hu E-D. A systematic review and a dose-response meta-analysis of coffee dose and nonalcoholic fatty liver disease. Clinical Nutrition; 2018.

19. Veronese N, Notarnicola M, Cisternino A, Reddavide R, Inguaggiato R, Guerra $\checkmark$, et al. Coffee intake and liver steatosis: a population study in a mediterranean area. Nutrients. 2018;10(1):89. https://doi.org/10.3390/nu1001 0089.

20. Chen S, Teoh NC, Chitturi S, Farrell GC. Coffee and non-alcoholic fatty liver disease: brewing evidence for hepatoprotection? J Gastroenterol Hepatol. 2014;29(3):435-41. https://doi.org/10.1111/jgh.12422.

21. Marventano S, Salomone F, Godos J, Pluchinotta F, Del Rio D, Mistretta A, et al. Coffee and tea consumption in relation with non-alcoholic fatty liver and metabolic syndrome: a systematic review and meta-analysis of observational studies. Clin Nutr. 2016;35(6):1269-81. https://doi.org/10.1016/ j.clnu.2016.03.012.

22. Noordzij M, Uiterwaal CS, Arends LR, Kok FJ, Grobbee DE, Geleijnse JM. Blood pressure response to chronic intake of coffee and caffeine: a metaanalysis of randomized controlled trials. LWW; 2005.

23. Steffen M, Kuhle C, Hensrud D, Erwin PJ, Murad MH. The effect of coffee consumption on blood pressure and the development of hypertension: a systematic review and meta-analysis. J Hypertens. 2012;30(12):2245-54. https://doi.org/10.1097/HJH.0b013e3283588d73.
24. Zhang Z, Hu G, Caballero B, Appel L, Chen L. Habitual coffee consumption and risk of hypertension: a systematic review and meta-analysis of prospective observational studies. Am J Clin Nutr. 2011;93(6):1212-9. https:// doi.org/10.3945/ajcn.110.004044.

25. D'Elia L, La Fata E, Galletti F, Scalfi L, Strazzullo P. Coffee consumption and risk of hypertension: a dose-response meta-analysis of prospective studies. Eur J Nutr. 2019;58(1):271-80. https://doi.org/10.1007/s00394-017-1591-z.

26. Xie C, Cui L, Zhu J, Wang K, Sun N, Sun C. Coffee consumption and risk of hypertension: a systematic review and dose-response meta-analysis of cohort studies. J Hum Hypertens. 2018;32(2):83-93. https://doi.org/10.1038/ s41371-017-0007-0.

27. Wedick NM, Brennan AM, Sun Q, Hu FB, Mantzoros CS, van Dam RM. Effects of caffeinated and decaffeinated coffee on biological risk factors for type 2 diabetes: a randomized controlled trial. Nutr J. 2011;10(1):93. https://doi. org/10.1186/1475-2891-10-93.

28. Kempf K, Herder C, Erlund I, Kolb H, Martin S, Carstensen M, et al. Effects of coffee consumption on subclinical inflammation and other risk factors for type 2 diabetes: a clinical trial. Am J Clin Nutr. 2010;91(4):950-7. https://doi. org/10.3945/ajcn.2009.28548.

29. Corrêa TA, Rogero MM, Mioto BM, Tarasoutchi D, Tuda VL, César LA, et al. Filtered coffee increases cholesterol and inflammation biomarkers independent of roasting degree: a clinical trial. Nutrition. 2013;29(7-8):97781. https://doi.org/10.1016/j.nut.2013.01.003.

30. Paiva C, Beserra B, Reis C, Dorea J, Da Costa T, Amato A. Consumption of coffee or caffeine and serum concentration of inflammatory markers: a systematic review. Crit Rev Food Sci Nutr. 2019;59(4):652-63. https://doi. org/10.1080/10408398.2017.1386159.

31. Zhang Y, Zhang D-Z. Associations of coffee consumption with circulating level of adiponectin and leptin. A meta-analysis of observational studies. Int J Food Sci Nutr. 2018;69(8):1003-12. https://doi.org/10.1080/09637486.201 8.1445202.

32. Williams CJ, Fargnoli JL, Hwang JJ, Van Dam RM, Blackburn GL, Hu FB, et al. Coffee consumption is associated with higher plasma adiponectin concentrations in women with or without type 2 diabetes: a prospective cohort study. Diabetes Care. 2008;31(3):504-7. https://doi.org/10.2337/ dc07-1952.

33. Rebello SA, Chen CH, Naidoo N, Xu W, Lee J, Chia KS, et al. Coffee and tea consumption in relation to inflammation and basal glucose metabolism in a multi-ethnic Asian population: a cross-sectional study. Nutr J. 2011;10(1):61. https://doi.org/10.1186/1475-2891-10-61.

34. Shahmohammadi HA, Hosseini SA, Hajiani E, Malehi AS, Alipour M. Effects of green coffee bean extract supplementation on patients with non-alcoholic fatty liver disease: a randomized clinical trial. Hepatitis Monthly. 2017;17(4).

35. Agudelo-Ochoa GM, Pulgarín-Zapata IC, Velásquez-Rodriguez CM, DuqueRamírez M, Naranjo-Cano M, Quintero-Ortiz MM, et al. Coffee consumption increases the antioxidant capacity of plasma and has no effect on the lipid profile or vascular function in healthy adults in a randomized controlled trial. J Nutr. 2016;146(3):524-31. https://doi.org/10.3945/jn.115.224774.

36. Rustenbeck I, Lier-Glaubitz V, Willenborg M, Eggert F, Engelhardt U, Jörns $A$. Effect of chronic coffee consumption on weight gain and glycaemia in a mouse model of obesity and type 2 diabetes. Nutr Diabetes. 2014;4(6):e123. https://doi.org/10.1038/nutd.2014.19.

37. MacKenzie T, Comi R, Sluss P, Keisari R, Manwar S, Kim J, et al. Metabolic and hormonal effects of caffeine: randomized, double-blind, placebocontrolled crossover trial. Metabolism. 2007;56(12):1694-8. https://doi.org/1 0.1016/j.metabol.2007.07.013.

38. van Dam RM, Pasman WJ, Verhoef P. Effects of coffee consumption on fasting blood glucose and insulin concentrations: randomized controlled trials in healthy volunteers. Diabetes Care. 2004;27(12):2990-2. https://doi. org/10.2337/diacare.27.12.2990.

39. Rakvaag E, Dragsted LO. Acute effects of light and dark roasted coffee on glucose tolerance: a randomized, controlled crossover trial in healthy volunteers. Eur J Nutr. 2016;55(7):2221-30. https://doi.org/10.1007/s00394-015-1032-9.

40. Shi $X$, Xue W, Liang S, Zhao J, Zhang X. Acute caffeine ingestion reduces insulin sensitivity in healthy subjects: a systematic review and meta-analysis. Nutr J. 2016;15(1):103. https://doi.org/10.1186/s12937-016-0220-7.

41. da Silva LA, Wouk J, Weber V, Eltchechem C, de Almeida P, Martins JCL. Mechanisms and biological effects of caffeine on substrate metabolism homeostasis: a systematic review. J Appl Pharm Sci. 2017;7:215.

42. Bruton JD, Lemmens $R$, Shi C-L, PERSSON-SJÖGREN S, WESTERBLAD H, Ahmed $M$, et al. Ryanodine receptors of pancreatic $\beta$-cells mediate a 
distinct context-dependent signal for insulin secretion. FASEB J. 2003;17(2): 301-3. https://doi.org/10.1096/fj.02-0481fje.

43. Jee SH, He J, Appel LJ, Whelton PK, Suh I, Klag MJ. Coffee consumption and serum lipids: a meta-analysis of randomized controlled clinical trials. Am 」 Epidemiol. 2001;153(4):353-62. https://doi.org/10.1093/aje/153.4.353.

44. d'Amicis A, Scaccini C, Tomassi G, Anaclerio M, Stornelli R, Bernini A. Italian style brewed coffee: effect on serum cholesterol in young men. Int J Epidemiol. 1996;25(3):513-20. https://doi.org/10.1093/ije/25.3.513.

45. Burr ML, Limb E, Sweetnam PM, Fehily A, Amarah L, Hutchings A. Instant coffee and cholesterol: a randomised controlled trial. Eur I Clin Nutr (United Kingdom). 1995

46. Cai L, Ma D, Zhang Y, Liu Z, Wang P. The effect of coffee consumption on serum lipids: a meta-analysis of randomized controlled trials. Eur J Clin Nutr. 2012;66(8):872-7. https://doi.org/10.1038/ejcn.2012.68.

47. Yukawa G, Mune M, Otani $H_{\text {, Tone }}$, Liang X-M, Iwahashi $\mathrm{H}$, et al. Effects of coffee consumption on oxidative susceptibility of low-density lipoproteins and serum lipid levels in humans. Biochem Mosc. 2004;69(1):70-4. https:// doi.org/10.1023/B:BIRY.0000016354.05438.0f.

48. Silvério ASD, Pereira RGFA, Lima AR, de Araújo Paula FB, Rodrigues MR, Baldissera $L$, et al. The effects of the decaffeination of coffee samples on platelet aggregation in hyperlipidemic rats. Plant Foods Hum Nutr. 2013; 68(3):268-73. https://doi.org/10.1007/s11130-013-0365-x.

49. Grioni S, Agnoli C, Sieri S, Pala V, Ricceri F, Masala G, et al. Espresso coffee consumption and risk of coronary heart disease in a large Italian cohort. PLoS One. 2015;10(5):e0126550. https://doi.org/10.1371/journal.pone.012 6550.

50. Wei M, Macera CA, Hornung CA, Blair SN. The impact of changes in coffee consumption on serum cholesterol. J Clin Epidemiol. 1995;48(10):1189-96. https://doi.org/10.1016/0895-4356(95)00023-W.

51. Shirlow MJ, Mathers CD. Caffeine consumption and serum cholesterol levels. Int J Epidemiol. 1984;13(4):422-7. https://doi.org/10.1093/ije/13.4.422.

52. Kobayashi-Hattori K, Mogi A, Matsumoto Y, Takita T. Effect of caffeine on the body fat and lipid metabolism of rats fed on a high-fat diet. Biosci Biotechnol Biochem. 2005;69(11):2219-23. https://doi.org/10.1271/bbb. 69.2219

\section{Publisher's Note}

Springer Nature remains neutral with regard to jurisdictional claims in published maps and institutional affiliations.

Ready to submit your research? Choose BMC and benefit from:

- fast, convenient online submission

- thorough peer review by experienced researchers in your field

- rapid publication on acceptance

- support for research data, including large and complex data types

- gold Open Access which fosters wider collaboration and increased citations

- maximum visibility for your research: over $100 \mathrm{M}$ website views per year

At BMC, research is always in progress.

Learn more biomedcentral.com/submissions 